\title{
The farther, the safer: a manifesto for securely navigating synthetic species away from the old living world
}

\author{
Philippe Marliere
}

Received: 21 May 2009/Revised: 5 August 2009/Accepted: 7 August 2009

(C) The Author(s) 2009. This article is published with open access at Springerlink.com

\begin{abstract}
Biotechnology has empirically established that it is easier to construct and evaluate variant genes and proteins than to account for the emergence and function of wild-type macromolecules. Systematizing this constructive approach, synthetic biology now promises to infer and assemble entirely novel genomes, cells and ecosystems. It is argued here that the theoretical and computational tools needed for this endeavor are missing altogether. However, such tools may not be required for diversifying organisms at the basic level of their chemical constitution by adding, substituting or removing elements and molecular components through directed evolution under selection. Most importantly, chemical diversification of life forms could be designed to block metabolic cross-feed and genetic crosstalk between synthetic and wild species and hence protect natural habitats and human health through novel types of containment.
\end{abstract}

Keywords GMO - Trophic containment .

Semantic containment $\cdot$ Xeno-nucleic acid

\section{A Renaissance for biology}

It has become customary to begin position papers on nanotechnology and synthetic biology with a visionary quote from Richard Feynman. For an essay on the guidance of genetic demiurges, no introduction seems more appropriate

P. Marliere $(\bowtie)$

Isthmus SARL, 75 rue de la Mare, 75020 Paris, France

e-mail: phmarliere@gmail.com than the oracle he once delivered at a teachers convention (Feynman 1968): "Science is the belief in the ignorance of experts."

History is replete with tragic and comic confrontations between experts fanatically attempting to enclose knowledge within a definitive representation of the world and discoverers stubbornly attempting to open, enlarge and disrupt it. This confrontational need to oppose dogmas and consecrated knowledge in order to achieve momentous discoveries did not always originate from improved hypotheses about the natural world. Certain major findings were not only made in spite of a misconception, sometimes they happened precisely because of it. Columbus would not have discovered the New World if he had not wrongly convinced himself that the actual size of the earth was smaller than had been rightly conjectured by Eratosthenes nearly 1,700 years earlier, and therefore that he could reach Japan from Spain after sailing only a few days beyond the western horizon (Boorstin 1985).

The knowledge that has been charted about the biological world encompasses metabolism, the genetic code and developmental regulation on one hand and ecological inventories, phylogeny and population genetics on the other. It does not cover the biotechnological innovations that industrial progress in agriculture, medicine, chemistry or energetics is calling for, as these innovations usually do not correspond to adaptive solutions that natural organisms have had to evolve. The art of navigation requires a knowledge of cartography, yet cannot be reduced to it, just as astronautics is distinct from astronomy. Likewise, the account of the living world afforded by molecular and evolutionary biology will be useful for exploring unnatural history but will not be sufficient for rationally designing life forms that did not occur or even could never have occurred during evolution. 
In a spirit akin to the Renaissance, biology is undergoing a metaphysical drama in which naturalists play the role of obscurantist experts and synthetic biologists that of heuristic conquistadores unhindered by their lack of navigation instruments in their haste to reach uncharted continents of life. The advent of a whole new biological world, which genetically modified organisms have only begun to incarnate, warrants the search for reliable methodologies to design and deploy artificial biodiversity while preserving the old natural world.

\section{Challenging expert beliefs}

To alleviate the fears that the public in numerous countries has expressed about genetically modified organisms (Torgersen 2004), the international community of experts in biology and biotechnology has adopted a non-transgressive stance about the operational scope and goals of genetic engineering. This stance reads essentially as two related beliefs: (i) the artificial biological objects created by human tinkering have remained within the close vicinity of the structures and functions at work in the natural living world; (ii) the immense innovative resource of genetic mutations, recombinations and interchanges in the wild vastly outnumbers, outsmarts and encompasses all our experimental attempts at reprogramming life. Both these beliefs should be amended, for the sake of science itself and also for the sake of preservation of the environment and human health.

The first expert belief (biological engineering explores only the neighborhood of natural biodiversity) is challenged by the demonstrated feasibility of generating viable descendants of common species whose chemical constitution deviates from those of all known extant species. Early experiments along this line were performed in the 50s and $60 \mathrm{~s}$, before the dogmas of molecular biology were carved in stone. They consisted of acclimatizing common cells to alien molecular components. Selenomethionine and trifluoroleucine were indeed shown to fully substitute for the universal amino acids methionine and leucine, respectively, in the proteins of Escherichia coli strains having lost the capability to make the corresponding natural amino acid (Cohen and Cowie 1957; Rennert and Anker 1963). Since apparently no mutation was required for enabling the usage of a synthetic ersatz, it is proper not to describe such changes as adaptations but as acclimatizations.

Even earlier, an alien analog of the vitamin biotin, oxabiotin, was shown to viably supplement certain bacterial strains lacking the ability to synthesize biotin (Pilgrim et al. 1945). Although such experiments did not correspond to genetic engineering, they demonstrated unequivocally that synthetic compounds absent from natural species could nevertheless be used by living cells as indispensible nutrients. Strains fully substituted with alien components grew poorly and reverted happily to the usage of the canonical components, amino acids or vitamins. Now, what would happen if such substitution experiments, which were seldom followed up in later years (Wong 1983) and still beg for a systematic investigation (Bacher and Ellington 2001), were conducted for an indefinite duration in genetic isolation from extant species? Would any expert take the risk of asserting that no unprecedented form of life would emerge from populations grown by substituting components of living cells with alien ersatz, all the more if several such substitutions were combined?

The second expert belief (molecular innovations of artificially engineered organisms are within evolutionary reach of natural biodiversity) is commonly evoked to minimize the hazards of GMO constructs as compared to "natural genetic engineering" (Shapiro 2005). The faith in the omnipotence of "natural genetic engineering" also exudes from almost every expert report on the seemingly inexhaustible emergence and inexorable dissemination of antibiotic resistance. All such beliefs are challenged by microbial demographics as inferred from ecology and geochemistry. Of the order of $10^{31}$ microbial genomes are presently at work at the earth's surface (Whitman et al. 1998), embodying most of the molecular know-how, metabolic and informational, that spontaneously originated, accumulated and integrated during evolution. Assuming boldly that each microbial cell undergoes $10^{4}$ generations per year (corresponding to a doubling time of $53 \mathrm{~min}$ ) and that microbial populations of constant size have been proliferating at the same rate for the past $410^{9}$ years, an upper-bound of $10^{45}$ genomes can be estimated to have been generated since the origin of cellular genomes on earth. Considering further that about one genome in a hundred differs by mutation (Drake et al. 1998) and that a self-sustaining cell's genome contains a minimum of one million bases, genotypes varying by at most 4-5 concomitant mutations are exhaustively screened all over the biosphere. Even if we account in our crude calculation for the contribution of conjugative plasmids and viral genomes (Ochman et al. 2000; Bubanovic et al. 2005) as well as transient hypermutator regimes (Ninio 1991), the conclusion cannot be avoided that evolution has so far explored only an extremely narrow sample of possible genotypes, recorded as combinations of $\mathrm{A}, \mathrm{C}, \mathrm{G}$ and $\mathrm{T}(\mathrm{U})$, and of phenotypes, recorded as metabolic routes, including DNA, RNA and protein synthesis.

The fact that extant biodiversity lacks some functional molecular device of atypical design in no way implies that this device has been already evaluated and rejected during evolution, but rather suggests that it was missed and remained inaccessible throughout natural history. 


\section{Upgrading and downgrading life forms}

In vitro selection of novel genetic sequences encoding RNA or proteins from random synthetic pools (Keefe and Szostak 2001) can be expected to provide completely unprecedented molecular lineages (Luisi et al. 2006), from which unprecedented functions could in principle be recruited in vivo. This feat, not yet accomplished, is a very promising source of artificial biodiversity to upgrade natural species. It would amount to applying at the most basic level the scenario of exaptation, i.e., "a feature that performs a function but that was not produced by natural selection for its current use" (Gould and Vrba 1982). Exaptation in man-remodeled living organisms can be anticipated to bring radical innovation if it is conducted in population enclaves, in other words if evolutionary intermediates are protected from predation by and competition with natural species in long-term cultures of clonal populations or ecosystems assembled from cooperating clones (Marliere et al. 2003).

It is a central tenet of Darwinian evolution that survival of the fittest leads to extinction of any variant bearing a trait that lowers survival or reproduction rate to the slightest extent relative to competitors lacking this trait, even if such a counter-selected trait would provide an overwhelming selective advantage at a further stage of functional elaboration. Natural selection should therefore be seen as routinely eliminating innovative features which would supersede current forms of life, if only these features would emerge as optimally crafted from scratch. To any educated computer scientist, the Origin of Species (Darwin 1859) just reads like a mechanistically sophisticated and brilliantly ornate description of a greedy algorithm, i.e., a procedure that iteratively optimizes a combinatorial configuration by choosing at the next step the variants of the prior configuration with equal or higher merit. Greedy algorithms are well known for bringing poor optimization solutions to most combinatorial problems (Bang-Jensen et al. 2004), which leads to the vexing notion that the whole process of spontaneous biological creation and evolution gave rise to the adoption of imperfect molecular devices and processes.

Would any expert thus take the risk of asserting that a common cell cannot be evolutionarily upgraded, say in a matter of one to ten thousand generations after insertion of properly designed synthetic genes (Jiang et al. 2008), so as to outcompete the wild species from which it originated ? If such could indeed be the case, should widespread marine bacteria such as species of the genus Pelagibacter or Prochlorococcus, which respectively release and fix most of the oceanic $\mathrm{CO}_{2}$, be excluded by synthetic biologists from experimental upgrading tests for preventing global climatic changes?
On the other hand, foundling genes originating from random sequence pools could possibly be mobilized to evolve awkward enzymes and replace efficient metabolic pathways with costly reaction itineraries, so as to downgrade their cellular hosts and make them inherently unable to outcompete their wild progenitors, i.e., built-in evolutionary losers in the struggle for life. The word "Verschlimmbesserung," which is used in German for mocking improvements that actually lower technical efficiency, could perhaps be used for designating the branch of synthetic biology aiming to protect natural ecosystems from artificial invaders through virtuous sabotage.

Combining the substitution of canonical cell components with alien ingredients (Cohen and Cowie 1957), the recruitment of novel genes with no biological ancestry from random pools (Keefe and Szostak 2001) or from molecular design (Jiang et al. 2008), and the long-term evolution of vast cell populations in isolation (Marliere et al. 2003) might now provide a potent methodology to explore a new biological world. Genesis of a second nature could turn out to be a rather cheap experimental process, at least in the microbiological realm. Before us likely lies the deployment of earth-bound exobiology, in other words the embodiment of virtual life forms and biological processes of metabolism and coding that did not occur or could not have evolved spontaneously through natural selection alone.

Let us now turn to the opportunities that such an exploratory endeavor provides to protect human health and preserve natural environments.

\section{The farther, the safer}

The word "cabotage" was coined by Spanish seamen to describe the safe navigation from cape to cape, in contrast to perilously sailing away toward open seas. Metaphorically, it is by restricting itself to exploring the vicinity of the known continent of life and staying near the shores and capes, the latter perhaps represented by extremophilic organisms, that biotechnology keeps steering evolutionary trajectories that do not depart from known forms of life. But the crux of the matter is that, as compared with the risk for navigation at sea, the risk for directed evolution of synthetic species is precisely the opposite, namely, the farther is now the safer. Indeed, the prospect of crafting and evolving the most deviant life forms as the safest way of establishing reliable and sustainable sources of chemicals, energy, not to mention nutrients, horresco referens, is only an apparent paradox.

The expert community and the lay public are still far from being convinced, but for opposite reasons. The experts of naturalist faith hold that current knowledge 
precludes the possibility of rapidly generating deviant forms of life because they worship natural biodiversity which they endow with the potency to have already screened all conceivable chemical constitutions and selected the optimal forms of life (Dryden et al. 2008). Certainly, this hypothesis will not survive confrontation with physical laws and computational principles. The lay public, following a consistent philosophical tradition (Heidegger 2000) and immemorial cosmological myths (Miller 1985), holds that human artifacts, including GMOs as their most transgressive avatars, have the inherent and fatal character of destroying natural harmony. The historical record of industrial and agricultural misdeeds gives credence to this hypothesis, although numerous innovations that have lessened the environmental impact of global practices while concomitantly improving industrial yields are grossly overlooked (Collins 2001).

A compromise measure reached by the lay and expert opinions has thus been to adopt the principle of physical containment for exploring artificial biodiversity and conduct reprogramming of genomes by gene transfer. So far, only the confinement of genetically reprogrammed plants has been discussed during the debates and is now legally enforced by some nations (Sparrow 2009). The experts see this measure as a futile tribute to superstition, while the public distrusts it as a mere decoy insufficient for averting genetic disaster and equivalent to the barriers erected for confining nuclear pollution, which were proven faulty at Chernobyl. Since the release of a single self-reproducing entity must be rendered impossible, there is no alternative to implementing fail-safe and leak-proof compartments that will passively block evasion of every individual cell or virus, including cases of negligent or malevolent human behavior. Nevertheless, no scientific group involved in synthetic biology is currently promoting the construction of heavy-duty facilities for confining synthetic organisms, as was done for the handling of highly infectious and deadly pathogens for medical research and presumably for the secret elaboration of biological weapons.

What makes infectious pathogens so dangerous to us, their prey and hosts, is their propensity to exploit us as a source of nutrients and energy, and hence ultimately our common chemical constitution with them. What renders us so vulnerable to insidious agents such as AIDS retroviruses and mad-cow prions, which can cross species boundaries and infect our cells, is our intimate sharing of the same coding system for nucleic acid and protein biosynthesis. What wild wolves have to fear from humans is no longer hunting and physical extermination, but out-breeding and genetic pollution by the reservoir of dog alleles which accumulated throughout domestication (e.g., drooping ears), thus exemplifying yet another modality of artificial molecular proximity as a threat to natural integrity (Butler
1994). Therefore, the surest if not simplest way to avoid risks of dissemination and contamination by potentially harmful synthetic species will be to evolve chemical constitutions as deviant as possible from that of natural species, and to rely on the persistence of these constitutions as a built-in measure for counteracting the colonization of wild habitats, including the human body.

Two categories of built-in safety appear tractable, trophic containment, for preventing metabolic cross-feed, and semantic containment, for preventing genetic cross-talk. The two categories can be combined and implemented redundantly in a single synthetic species.

\section{Trophic containment}

Trophic containment consists of making the proliferation of a species dependent sine qua non upon the exogenous supply of an alien molecule that does not occur in food chains of natural habitats in the broadest sense, not as a geochemical ingredient, nor as a regular chemical component of cells nor as a by- or side-product of regular metabolites. Trophic containment features the advantage of a stoichiometric requirement: a population of the synthetic species requiring a xeno-nutrient will reach a size proportional to the quantity of that alien compound made available to it. Sufficient sophistication will have to be introduced in this scheme so that the requirement for the xeno-nutrient cannot be circumvented by the synthetic organism through utilization of a surrogate molecule present in food chains, nor through spontaneous discovery of a biosynthetic pathway for the xeno-nutrient by evolving enzymes and metabolic pathways.

The evolution of metabolic processes in wild species for degrading the xeno-nutrient and using it as source of elements and energy poses no problem. It could even be considered as an advantage since biodegradation by wild species will lower the amount of available xeno-nutrient and therefore the population size of synthetic species dependent upon its exogenous supply. The scientific literature bears mention of a "microbial infallibility hypothesis," according to which some micro-organism will be found to degrade every molecule that is made by any living organism (Gale 1952). Few organic molecules seem to accumulate in natural habitats, which implies that almost all of them must therefore be somehow recycled, even the crosslinked polymers of lignin, which concentrate in the humus of forest ground to be eventually volatilized by heterotrophs such as fungi. The biodegradability of synthetic molecules such as pesticides and herbicides, polymers such as nylon, and even artificial antibiotics, lends further credence to this hypothesis: after a latency in the order of a decade or two, microbial species able to use 
them as nutrients begin to emerge and the genetic trait of degradative capability spreads by "interspecific horizontal transfer." Note that the progressive acquisition of degradative reactions for converting a xeno-nutrient into common cellular chemicals (metabolites) by natural microbes does not imply that synthetic reactions for producing this xeno-nutrient could be progressively acquired by the reprogrammed species or by natural species adapting to undergo nutritional or genetic exchanges with artificial species. Indeed, reactions with water and with oxygen are used by scavenger micro-organisms to dismember organic molecules but not for assembling them, because these degradative reactions are totally irreversible.

By introducing chemical motifs that are very rare among natural biomolecules (e.g., ether bridges, polycyclic aliphatic groups) or involving elements absent from natural biomatter and highly improbable in aqueous environments (e.g., silicon-carbon bonds), xeno-nutrients could be designed so as to impede their biosynthetic access. In addition, dependency on several xeno-nutrients inaccessible from existing metabolism and food chains could be combined to saturate the search through natural selection for producing these compounds autonomously in synthetic species.

The three types of xeno-nutrients that seem more suitable for addicting synthetic species would be amino acids (the building blocks of proteins), nucleotides (the building blocks of nucleic acids) and coenzymes (vitamins, the sophisticated chemical catalysts of certain metabolic reactions). Lipids (the constituents of membranes) and sugars (the building blocks of cell surfaces and cell walls), whose structures vary much throughout species and even under different growth conditions for a given species, appear less strategic for diversifying the chemical arsenal of life. Methods are available for mobilizing additional amino acids in proteins of reprogrammed bacteria (Lemeignan et al. 1993; Mehl et al. 2003). It remains to be proven, however, that such constructs with an augmented genetic code can be stably bred in the long run.

Synthetic coenzymes that would not replace, as does oxabiotin for biotin (Pilgrim et al. 1945), but add a catalytic capability for performing metabolic reactions absent from natural cells, hence enabling colonization of certain industrial habitats, represent a tantalizing option for enforcing trophic containment. Elaboration of such xenovitamins should become a flourishing branch of synthetic biology.

\section{Xeno-nucleic acids}

Though more difficult to tackle, the addiction of synthetic organisms to additional nucleotides deserves a special interest because it would open a path toward a radically novel class of GMOs rendered safe through semantic as well as trophic containment (Herdewijn and Marliere 2009).

Each class of the natural nucleic acids, DNA and RNA, convey genetic information under the form of specific successions of nucleobases chosen among four types forming two pairs A:T and G:C (in RNA, T is slightly altered into $\mathrm{U}$ ). Each base A, C, G and T (U) is attached to a backbone motif, of which there are two types, ribose in RNA and deoxyribose in DNA. Synthetic alternatives of both nucleobases and backbone motifs have been demonstrated to lead to well-behaved unnatural nucleic acids when incorporated into polymers (Sismour and Benner 2005). For the sake of clarity, it is worth dwelling on the potential biological consequences of the usage of these two broad classes of unnatural nucleic acid building-blocks.

Nucleotides bearing additional bases, say V and W, on a canonical DNA or RNA backbone and able to form pairs $\mathrm{V}: \mathrm{W}$ compatible with $\mathrm{A}: \mathrm{T}$ and $\mathrm{G}: \mathrm{C}$ could in principle progressively invade existing DNA and RNA molecules and provide a larger lexicon of sequence combinations, e.g., VWV or CWA, that natural species would be unable to propagate. The technological sophistication needed for equipping the genetic expression system of a synthetic organism with an additional pair of complementary bases ( $\mathrm{V}$ and $\mathrm{W}$ ) would amount to supplying four metabolites (dVTP, dWTP, rVTP, rWTP) and at least two catalytic macromolecules (one altered tRNA and one altered mRNA) in order to become operational in conveying genetic information into proteins (Bain et al. 1992). At least three additional and reliable base-pair systems have been validated in vitro and await launching in vivo (Sismour and Benner 2005; Yang et al. 2007; Kimoto et al. 2008).

A different scheme features additional nucleotides bearing canonical bases onto a chemically deviant backbone in lieu of ribose and deoxyribose (Pochet et al. 2003). The polymerization of such deviant building blocks would not result into the invasion of existing DNA or RNA polymers, but into the generation of a distinct type of informational biopolymer, XNA (xeno-nucleic acid). XNA would be propagated as a chemically estranged form of plasmid, that should not interfere with DNA or RNA biosynthesis or function (Herdewijn and Marliere 2009). The technological sophistication needed for implementing this scheme would amount to supplying at least one additional pair of metabolites (e.g., xATP and xUTP) and two specialized polymerization enzymes (an XNA-dependent XNA polymerase and an XNA-dependent RNA polymerase). The polymerization of three deviant backbones is currently being pursued in vitro (Kempeneers et al. 2005; Tsai et al. 2007; Schmidt 2009), and a consortium, 
coordinated by Piet Herdewijn in Leuven, has formed with the aim to launch an XNA bridgehead in vivo (http://www. synbiosafe.eu/index $=$ php? page $=$ orthosome).

Safe vector-host systems and prevention of carry-over contamination in PCR are commonly implemented using natural molecular mechanisms (Glenting and Wessels 2005) such as toxin/antitoxin neutralization (Stieber et al. 2008) and repair by uracil-N-glycosylase (Longo et al. 1990). Their high efficiency of built-in inactivation can be considered as satisfactory in the laboratory, yet they all give rise to rare escape events and thus fall short of the criterion of infallibility required for protecting human health and wild habitats. It is this absolute criterion of dependency on the supply of xeno-nutrients that propagation of XNA-supported information will have to pass for qualifying as a safe GMO technology.

\section{Semantic containment}

In principle, semantic containment could be enforced without requiring any additional or alternative buildingblock, by reassigning codons between canonical amino acids. There exist 64 different triplets of the four canonical bases A, C, G, U, serving as codons for the 20 canonical amino acids and punctuation signals in protein synthesis on the ribosome. Highly discriminating enzymes (synthetases) attach a given amino acid to a cognate RNA adaptor (tRNA) to generate the aminoacyl-tRNAs (aa-tRNA) that are condensed into proteins (amino acid polymers) in response to codon signals on messenger RNA (mRNA), thus freeing tRNAs again to enter another condensation cycle. The overall process, called genetic translation, which involves several hundred RNA and protein molecules and stands as the most sophisticated function evolved by life, originated nearly 4 billion years ago and has remained nearly invariant since (Ambrogelly et al. 2007). Few extant species deviate in the assignment of triplet nucleotide codons to amino acids, an empirical observation that was dogmatized as the Universal Genetic Code. It has been argued that this (nearly) universal conservation was not dictated by physical and chemical constraints, but adopted as an indirect consequence of interspecific genetic transfers (Syvanen 1985). Any species deviating from the genetic "lingua franca" spoken by other species would indeed find itself cut off from the rest of the living world and could not benefit from the know-how elaborated abroad (Ambrogelly et al. 2007).

Creating genetic enclaves by actively enforcing codon reassignments in cell lineages thus appears as a straightforward scenario for progressively alienating synthetic organisms and building a Tower of Babel of genetic codes. Whole rewriting of bacterial genomes could be used for this purpose, but alternative methods based on long-term cultivation in isolation of missense strains have also undergone a proof a concept (Döring and Marlière 1998). A missense strain is a population of microbial cells in which non-cognate chemical linkages between an amino acid and a tRNA occurs (non-canonical aa-tRNA) through the mobilization of mutant tRNA or mutant synthetase. Certain wild bacterial species with deviant DNA composition, such as $\mathrm{A}+\mathrm{T}$ rich Mycoplasma and $\mathrm{G}+\mathrm{C}$ rich Micrococcus no longer use certain codons, which are thus available for reassignment to canonical or synthetic amino acids. Such "compositional extremophiles" offer a precious starting point with a vast potential for reprogramming and breeding synthetic organisms unable to interpret the genes from natural species or the genes from other separately estranged lineages.

In principle, this approach of genetic estrangement through codon reassignment can be freely combined with the nutritional enforcement of additional building blocks, amino acids, coenzymes and nucleotides, as described above. Since estranging the genetic code and enforcing alien nutrients can in turn each be multiplied in a given cell, a workable approach should attempt to simultaneously and stably prevent cross-talk and cross-feed between artificial and natural biodiversities. Such a scenario of redundant semantic and trophic containment (RST), which remains to be validated operationally, provides a conceptual basis for establishing a constructive debate with environmentalists about the preservation of natural habitats.

\section{Humble programmers and modest creators}

Aside from the numerous pioneering demonstrations that augur most favorably for the advances to come, many of the prophecies recently uttered in the budding field of synthetic biology are pervaded by wishful thinking (Pollack 2006). The risk to overthrow the formal criterions of scientific discourse is of particular concern in North America, where the souls of the synthetic biology community at times seem to be possessed by the demon of hype. Indeed, claiming that the capability to chemically synthesize entire microbial genomes, and soon after entire cells, is about to solve the global energetic equation by enabling the metabolic supply of an inexhaustible amount of hydrogen from sunlight and seawater, borders on shamanic delusion (Shand et al. 2007). This sounds like claiming that the capability to assemble a pipe organ also endows the organ builder with the gift of composing toccatas and fugues by sheer improvisation. Of course, such utterances do not diminish in the least the potential of reprogramming cells with whole synthetic genomes for 
scientific and industrial purposes. However, for realizing its promises, an explicit programming of phenotypes by rewriting genotypes will need to be conducted, the principles of which still remain in limbo.

Also inflated with wishful thinking seems to be the claimed potency of standard biomolecular bricks, chassis, foundries, registries and the like, for paralleling in biotechnology the design and production of industrial artifacts such as the integrated circuits of electronics (Shand et al. 2007). Alas, self-reproduction and evolvability are not just features that can be added to the list of other technical specifications, because they constitute an undefined dynamic challenge that precisely forbids the application of the methods of design and implementation that engineers have practiced so far. A suggestive metaphor pictures evolution as modifying the wings and reactors of an airplane in-flight. Methods for abstracting such unprecedented technological requirements are still cruelly lacking. It should be recalled in this respect that the founding fathers of informatics found it wise early on to proscribe selfmodifying programs altogether.

Clearly, the simple-minded modular formats of hierarchical design which are currently heralded to make synthetic biology comply with the imperatives of engineering (Endy 2005) will be insufficient to change the chemical constitution of synthetic organisms as sketched above. No college of living experts or convention of expert systems will for some time predict the correct text to write in synthetic genomes in order to make, for example, informational biopolymers and metabolism accrued by incorporating the element silicon or devoid of the element phosphorus (Westheimer 1987), though such constructs should presumably be viable if not accessible from extant forms of life. It is in tackling the most arduous compositional problems, by adding, substituting or removing types of molecular components or chemical elements in living organisms (Walter et al. 2005) that we shall learn the methods needed by the "modest creator" to provide safe solutions that pass the innocuousness test in ecosystems just as the "humble programmer" has to learn methods to pass the optimality test of algorithms in computers (Dijkstra 1972). In so doing, synthetic biology will follow the precepts that were put forward by Edsger Dijkstra, arch-formalist of computation and founder of hierarchical design and levels of abstraction, which are precisely invoked for empowering synthetic biology (Endy 2005).

Less predisposed to succumb to hype, the European community of synthetic biologists nevertheless suffers from its own frailties, institutional conformism and pusillanimity. Europeans should always remember that they are the descendants of those who did not leave to conquer the New World. It has been argued that instead, they embarked on an inner journey where they found the abstraction of mechanisms and scientific laws (Sloterdijk 2001). Americans meanwhile resorted to technical pragmatism for accommodating a pristine continent. As an endeavor requiring both abstraction and pragmatism, synthetic biology offers a conciliatory forum for "modest creators" from all cultures to shape the science of living systems, to forge the technology of safe and ever more varied forms of life and to seal the pact of ethical accountability that societies and their governments ask for, in exchange for the freedom to breed a second nature.

Acknowledgments I am grateful to Susan Cure and Peter Brooks for taming my rhetoric in English, to Dieter Söll for conspiring to subvert the genetic code, to Rupert Mutzel, Volker Döring and Markus Schmidt for sharpening my evolutionary arguments, and to Piet Herdewijn and Don Hilvert for blessing my schemes with chemical consistency whenever it was needed. Preparation of this manuscript was supported by a grant from the Fonds zur Förderung der wissenschaftlichen Forschung (FWF, the Austrian Science Fund), project I215-B17, "Investigating the biosafety and risk assessment needs of synthetic biology in Austria (Europe) and China."

Open Access This article is distributed under the terms of the Creative Commons Attribution Noncommercial License which permits any noncommercial use, distribution, and reproduction in any medium, provided the original author(s) and source are credited.

\section{References}

Ambrogelly A, Palioura S, Söll D (2007) Natural expansion of the genetic code. Nat Chem Biol 3:29-35

Bacher JM, Ellington AD (2001) Selection and characterization of Escherichia coli variants capable of growth on an otherwise toxic tryptophan analogue. J Bacteriol 183:5414-5425

Bain JD, Switzer C, Chamberlin AR, Benner SA (1992) Ribosomemediated incorporation of a non-standard amino acid into a peptide through expansion of the genetic code. Nature 356: 537-539

Bang-Jensen J, Gutin G, Yeo A (2004) When the greedy algorithm fails. Discret Optim 1:121-127

Boorstin DJ (1985) The discoverers: a history of man's search to know his world and himself. Random House, New York, pp 224-234

Bubanovic I, Najman S, Andjelkovic Z (2005) Origin and evolution of viruses: escaped DNA/RNA sequences as evolutionary accelerators and natural biological weapons. Med Hypotheses 65:868-872

Butler D (1994) Bid to protect wolves from genetic pollution. Nature $370: 497$

Cohen GN, Cowie DB (1957) Remplacement total de la methionine par la selenomethionine dans les proteines d'Escherichia coli. C R Acad Sci 244:680-683

Collins T (2001) Toward sustainable chemistry. Science 291:48-49

Darwin CR (1859) On the origin of species by means of natural selection, or the preservation of favoured races in the struggle for life. http://www.talkorigins.org/faqs/origin.html

Dijkstra EW (1972) The humble programmer. EWD340. http:// www.cs.utexas.edu/users/EWD/transcriptions/EWD03xx/EWD3 40.html 
Döring D, Marlière P (1998) Reassigning cysteine in the genetic code of Escherichia coli. Genetics 150:543-551

Drake JW, Charlesworth B, Charlesworth D, Crow JF (1998) Rates of spontaneous mutation. Genetics 148:1667-1686

Dryden DTF, Thomson AR, White JH (2008) How much of protein sequence space has been explored by life on Earth? J R Soc Interface 5:953-956

Endy D (2005) Foundations for engineering biology. Nature 438: $449-453$

Feynman RP (1968) What is science? Phys Teach 7:313-320

Gale EF (1952) The chemical activities of bacteria. Academic Press, New York, p 5

Glenting J, Wessels S (2005) Ensuring safety of DNA vaccines. Microb Cell Fact 4:26

Gould SJ, Vrba ES (1982) Exaptation-a missing term in the science of form. Paleobiology 8:4-15

Heidegger M (2000) An introduction to metaphysics. Yale University Press, New Haven

Herdewijn P, Marliere P (2009) Toward safe genetically modified organisms through the chemical diversification of nucleic acids. Chem Biodivers 6:791-808

Jiang L, Althoff EA, Clemente FR, Doyle L, Röthlisberger D, Zanghellini A, Gallaher JL, Betker JL, Tanaka F, Barbas CF III, Hilvert D, Houk KN, Stoddard BL, Baker D (2008) De novo computational design of retro-aldol enzymes. Science 319: $1387-1391$

Keefe AD, Szostak JW (2001) Functional proteins from a randomsequence library. Nature 410:715-718

Kempeneers V, Renders M, Froeyen M, Herdewijn P (2005) Investigation of the DNA-dependent cyclohexenyl nucleic acid polymerization and the cyclohexenyl nucleic acid-dependent DNA polymerization. Nucleic Acids Res 33:3828-3836

Kimoto M, Kawai R, Mitsui T, Yokoyama S, Hirao I (2008) Efficient PCR amplification by an unnatural base pair system. Nucleic Acids Symp Ser 2008(52):469-470

Lemeignan B, Sonigo P, Marliere P (1993) Phenotypic suppression by incorporation of an alien amino acid. J Mol Biol 231:161-166

Longo MC, Berninger MS, Hartley JL (1990) Use of uracil DNA glycosylase to control carry-over contamination in polymerase chain reactions. Gene 93:125-128

Luisi PL, Chiarabelli C, Stano P (2006) From the never born proteins to the minimal living cell: two projects in synthetic biology. Orig Life Evol Biosph 36:605-616

Marliere P, Mazel D, Mutzel R (2003) Method for obtaining cells with new properties. Patent WO03004656

Mehl RA, Anderson JC, Santoro SW, Wang L, Martin AB, King DS, Horn DM, Schultz PG (2003) Generation of a bacterium with a 21 amino acid genetic code. J Am Chem Soc 125:935-939

Miller J (1985) The vision of cosmic order in the vedas. Routledge \& Kegan, Paul, London

Ninio J (1991) Transient mutators: a semiquantitative analysis of the influence of translation and transcription errors on mutation rates. Genetics 129:957-962
Ochman H, Lawrence JG, Groisman EA (2000) Lateral gene transfer and the nature of bacterial innovation. Nature 405:299-304

Pilgrim FJ, Axelrod AE, Winnick T, Hofmann K (1945) The microbiological activity of an oxygen analog of biotin. Science 102:35-36

Pochet S, Kaminski PA, Van Aerschot A, Herdewijn P, Marlière P (2003) Replication of hexitol oligonucleotides as a prelude to the propagation of a third type of nucleic acid in vivo. C R Acad Sci Biol 326:1175-1184

Pollack A (2006) Custom-made microbes, at your service. The New York Times, 17 Jan 2006

Rennert OM, Anker HS (1963) On the incorporation of $5^{\prime}, 5^{\prime}, 5^{\prime}$ trifluoroleucine into proteins of E. coli. Biochemistry 2:471-476

Schmidt M (2009) Do I understand what I can create? Biosafety issues in synthetic biology. In: Schmidt M, Kelle A, Ganguli A, de Vriend $\mathrm{H}$ (eds) Synthetic biology the technoscience and its societal consequences, vol 6. Springer Academic Publishing, Berlin

Shand H, Thomas J, Wetter KJ (2007) Playing god. The Ecologist, 1 May 2007

Shapiro JA (2005) A 21st century view of evolution: genome system architecture, repetitive DNA, and natural genetic engineering. Gene 345:91-100

Sismour AM, Benner SA (2005) Synthetic biology. Expert Opin Biol Ther 5:1409-1414

Sloterdijk P (2001) Kränkung durch Maschinen. In: Sloterdijk P (ed) Nicht gerettet, Versuche nach Heidegger. Suhrkamp Verlag, Frankfurt, pp 338-366

Sparrow PA (2009) GM risk assessment. Methods Mol Biol 478:315330

Stieber D, Gabant P, Szpirer C (2008) The art of selective killing: plasmid toxin/antitoxin systems and their technological applications. Biotechniques 45:344-346

Syvanen M (1985) Cross-species gene transfer; implications for a new theory of evolution. J Theor Biol 112:333-343

Torgersen H (2004) The real and perceived risks of genetically modified organisms. EMBO Rep 5:S17-S21

Tsai CH, Chen J, Szostak JW (2007) Enzymatic synthesis of DNA on glycerol nucleic acid templates without stable duplex formation between product and template. Proc Natl Acad Sci USA 104:14598-14603

Walter KU, Vamvaca K, Hilvert D (2005) An active enzyme constructed from a 9-amino acid alphabet. J Biol Chem 280:37742-37746

Westheimer FH (1987) Why nature chose phosphates. Science 235:1173-1178

Whitman WB, Coleman DC, Wiebe WJ (1998) Prokaryotes: the unseen majority. Proc Natl Acad Sci USA 95:6578-6583

Wong JT (1983) Membership mutation of the genetic code: loss of fitness by tryptophan. Proc Natl Acad Sci USA 80:6303-6306

Yang Z, Sismour AM, Sheng P, Puskar NL, Benner SA (2007) Enzymatic incorporation of a third nucleobase pair. Nucleic Acids Res 35:4238-4249 